\title{
Was kommt mit dem «Rosalinde- Effekt» auf die Schweiz zu?
}

EMH News Service

\section{Bahnbrechende Studienresultate}

Ende Februar dieses Jahres - nach der Publikation bahnbrechender Resultate im Journal of Obscure Research in Obstetrics [1] - wurde in der Geburtsklinik Rosalinde (Vancouver, Kanada) gefeiert. In der Tat spiegeln die Resultate der Studie die grösste Errungenschaft in der Frauenheilkunde seit mehr als 60 Jahren wider - nach der Entwicklung der hormonellen Schwangerschaftsverhütung (der «Pille») in den 1950er Jahren. Die Schweizerische Ärztezeitung berichtete bereits ausführlich darüber [2]. Noch einmal kurz die wichtigsten Fakten: Die in der Rosalinde-Klinik tätige ärztliche Forschergruppe um Dr. Marc Cassari entdeckte 2004 das in der embryonalen Leber von Säugetieren gebildete Fetotropin. Daraufhin ging sie der Frage nach, ob sich durch eine

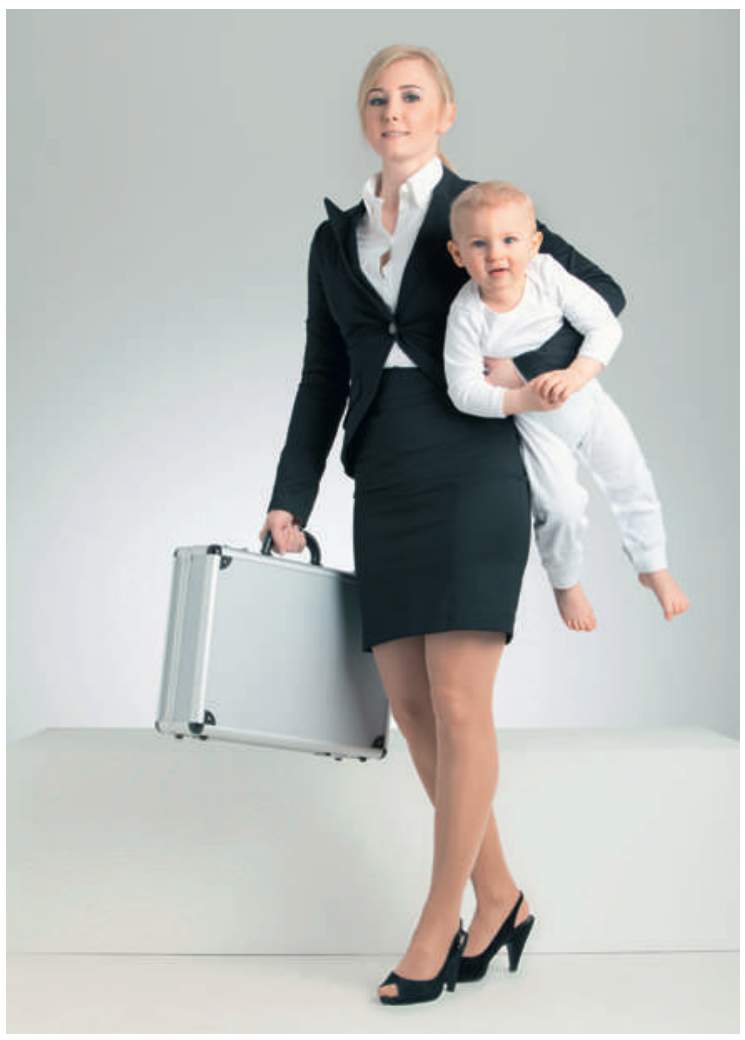

Besonders Geschäftsfrauen schätzen die verkürzte Schwangerschaft durch Fetotropin. zusätzliche Verabreichung des Wachstumsfaktors die natürliche Fetalreifung beeinflussen lässt. Nach einem Jahrzehnt ausgiebiger Grundlagenforschung konnte die Gruppe nun kürzlich zeigen, dass der Faktor auch beim Menschen das Fetalwachstum beschleunigt und die

\section{Achtzig Probandinnen brachten im} vergangenen Jahr ausgereifte und gesunde Kinder zur Welt.

Schwangerschaftsdauer um fast zwei Monate verkürzt. Er muss dazu zwischen der 11. und 13. Schwangerschaftswoche, also nach der Ausbildung der inneren Organe, in höheren Dosen maternal (subkutan) appliziert werden. Achtzig Probandinnen brachten im vergangenen Jahr ausgereifte und gesunde Kinder zur Welt (42 Mädchen, 38 Jungen). Die häufigsten Namen der Kinder (von dankbaren Eltern) sind Tropina bzw. Tropino.

\section{Befürworter und Gegner}

Die Synthese des bei der Schwangeren verabreichten $3 \beta$-Hydroxy-Fetotropin ist komplex und teuer; daher steht es gegenwärtig nur in geringen Mengen zur Verfügung. Zurzeit wird es ausschliesslich in der Rosalinde-Klinik im Rahmen von Studien verabreicht. Noch haben die kanadischen Ärzte die alleinigen Rechte für die Anwendung beim Menschen; doch im Hintergrund streiten sich bereits sechs Pharmakonzerne um die Vertriebsrechte von 3 $\beta$-HydroxyFetotropin, darunter zwei grosse schweizerische Unternehmen.

Die Feierlaune in der "Rosalinde» hat zwischenzeitig deutlich abgenommen. Heute gleicht die Geburtsklinik eher einer Festung. Tausende Frauen im ersten Schwangerschaftsdrittel begehren Einlass und wollen sich einer Fetotropin-Behandlung unterziehen; doch das Wach- und Sicherheitspersonal versucht sie daran zu hindern, Ärzte und Pflegekräfte stehen unter Polizeischutz. Um die Klinik herum hat sich eine Vielzahl von Fetotropin-Gegnern versammelt und demonstriert gegen die neue Methode zur Schwangerschafts- 


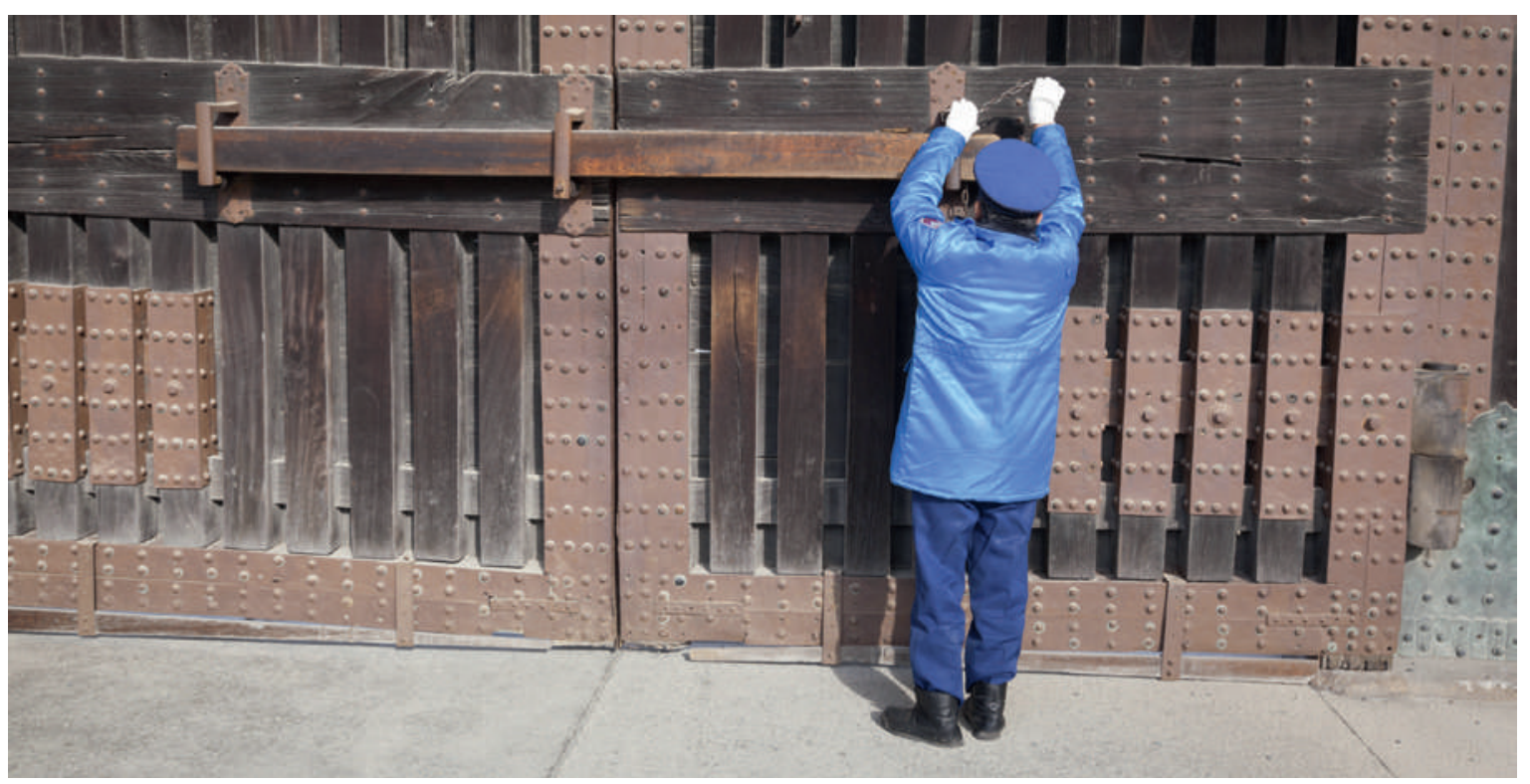

Elektronische Sicherheitssysteme leisten nach Ansicht der Rosalinde-Klinikleitung keinen ausreichenden Schutz vor Frauen, die zu allem entschlossen sind.

verkürzung. Eine Entspannung der Situation ist in den nächsten Wochen kaum zu erwarten.

\section{Viele ungelöste Fragen}

Die Meinungen über Fetotropin gehen weit auseinander. Die Frauen im gebärfähigen Alter sind gespalten. Die Fachgesellschaften, Frauenrechtsgruppen, Arbeitgeberverbände und die Canadian Airlines befürworten eine kontrollierte Zuteilung - wenn auch aus unterschiedlichen Gründen. Unter den Gegnern finden sich Vertreter aus dem kirchlichen Umfeld, der Appenzeller Kleinkinderschutzbund und Anbieter von Umstandsmode. Vieles dreht sich um die Frage: «Ist es richtig, in die Prozesse der Natur einzugreifen?» Dr. Marc Cassari hat sich dazu selbst am 1. April in einem Interview geäussert [3]: «Die Schwangerschaftsdauer stellt in der Natur keine konstante Grösse dar. Beim Goldhamster beträgt die Tragzeit 16 Tage, beim afrikanischen Elefanten 22 Monate. Der Mensch befindet sich dazwischen, da machen sieben oder neun Monate keinen bedeutenden Unterschied aus.»

Weniger umstritten ist die Anwendung bei Frauen, bei denen eine Schwangerschaft mit einem gesundheitlichen Risiko verbunden ist; mit der Verkürzung der Schwangerschaft könnten Komplikationen vermieden werden, insbesondere solche, die erst im achten oder neunten Schwangerschaftsmonat auftreten. Aber sind eine frühzeitige Rückkehr an den Arbeitsplatz oder die Verschleierung der Vaterschaft ethisch zu rechtfertigende Indikationen? Welche Kriterien wären überhaupt bei der Zuteilung einer begrenzten Fetotropin-Menge anzuwenden? Wer sollte zukünftig Fetotropin verabreichen dür- fen? Und sollte Fetotropin durch das Solidarsystem finanziert werden? Dies ist nur eine Auswahl von Fragen, die sich momentan stellen. Nicht auszuschliessen ist, dass durch die Verkürzung der Schwangerschaftsdauer mehr Frauen bereit sein könnten, ein Kind zu bekommen; die Fetotropin-Verabreichung weist somit auch eine nicht $\mathrm{zu}$ unterschätzende gesellschaftspolitische Dimension auf.

\section{Die Schweiz bereitet sich vor}

Die Schweiz ist dabei, sich auf den erwarteten «Rosalinde-Effekt» vorzubereiten - denn dass Fetotropin auch hier Einzug hält, scheint nur eine Frage der Zeit zu sein. In einigen Kliniken mit geburtshilflichen Abteilungen werden schon jetzt Stahltüren und Fenster aus Panzerglas eingebaut, geheime Zugänge und Fluchtwege angelegt. An die Nationale Ethikkommission erging der Auftrag, sich mit den ethischen Aspekten der Fetotropin-Gabe auseinanderzusetzen und Empfehlungen zu Händen des Bundesrats auszuarbeiten.

\section{Heute gleicht die Geburtsklinik eher einer Festung.}

Wie denkt die schweizerische Ärzteschaft über die Verabreichung von Fetotropin und die damit verbundene Verkürzung der Schwangerschaftsdauer? Uns interessiert Ihre Meinung! Nutzen Sie dazu das Kommentarfeld im Forum auf unserer Webseite. Wir bedanken uns vorab für Ihre zahlreichen Beiträge.

\section{Bildnachweis}

Geschäftsfrau: MEV-Verlag GmbH, Germany

Rosalinde-Tor: Anthony Brown | Dreamstime.com 